\title{
Static and dynamic effects of flicker in phase multilevel elements on LCoS devices
}

\author{
A. Márquez ${ }^{1,2, *}$, F. J. Martínez ${ }^{1,2}$, S. Gallego ${ }^{1,2}$, M. Ortuño ${ }^{1,2}$, J. Francés ${ }^{1,2}$, A. Beléndez ${ }^{1,2}$, I. \\ Pascual $^{2,3}$ \\ ${ }^{1}$ Dept. de Física, Ing. de Sistemas y T. Señal, Univ. de Alicante, Ap. 99, E-03080, Alicante, Spain \\ ${ }^{2}$ I.U. Física Aplicada a las Ciencias y las Tecnologías U. de Alicante, Ap. 99, E-03080, Alicante, Spain \\ ${ }^{3}$ Dept. de Óptica, Farmacología y Anatomía, Univ. de Alicante, Ap. 99, E-03080, Alicante, Spain
}

\begin{abstract}
Phase-only modulation is necessary in a great number of modern spatial light modulation applications, and the spatial light modulator (SLM) technology of choice is usually the parallel-aligned liquid crystal on silicon (PA-LCoS) microdisplay. Various degradation effects have been analyzed in the literature which may be introduced by SLMs and whose quantitative knowledge enables to select the best working conditions and/or to design specific compensation strategies to diminish negative effects. In this paper we concentrate on the phase flicker typically produced by PA-LCoS devices. The availability of a recent polarimetric-based method, the average Stokes polarimetric technique, to measure the linear retardance and its flicker amplitude eases the capability to simulate the performance of spatially varying phase multilevel elements typically addressed onto PA-LCoS devices. A representative element is the blazed grating. Recently we demonstrated the capability of the calibration provided by the average Stokes polarimetric technique to predict the performance of blazed gratings, both their average diffraction efficiency, static analysis, and its associated time fluctuation, dynamic analysis. In the present work we take advantage of the demonstrated predictive capability of our approach to analyse to find the wide range of applicability of PA-LCoS devices in applications in spite of flicker.
\end{abstract}

Keywords: Liquid crystal on silicon displays, parallel aligned, retardance measurement, phase-only modulation, spatial light modulation, flicker, diffractive optics, Stokes polarimetry.

\section{INTRODUCTION}

Spatial light modulators (SLM) are complex devices with a great importance in a large number of optical and photonic applications. Among the different SLM technologies probably the most widespread is the one based on liquid crystal devices, with the liquid crystal on silicon displays (LCoS) being one of the latest developments ${ }^{[1]-[3]}$. In most of the spatial light modulation applications, it is very appealing the possibility to implement phase-only modulation of the light wavefront. This is the case in diffractive optics ${ }^{[4]}$, optical storage $\mathrm{e}^{[5][6]}$, optical metrology ${ }^{[7]}$, reconfigurable interconnects ${ }^{[8][9]}$, wavefront sensing of structured light beams ${ }^{[10]}$, holographic optical traps ${ }^{[11]}$, or quantum optical computing $^{[12]}$. Parallel-aligned liquid crystal on silicon (PA-LCoS) microdisplays enable phase-only operation without coupled amplitude modulation, thus making them one the most appealing SLMs nowadays ${ }^{[1]}$.

PA-LCoS microdisplays can be assimilated to linear variable retarders being their performance characterized by their linear retardance as a function of the applied voltage. However, proper evaluation of these devices has shown that they exhibit fluctuations (flicker) in their retardance values ${ }^{[13]-[15]}$. Some methods have been proposed enabling to evaluate both the retardance and the flicker magnitudes ${ }^{[16]-[18]}$. In particular, we have demonstrated the predictive capability of a time-averaged Stokes polarimetric technique ${ }^{[18]}$, and we have analysed its robustness and its applicability to characterize PA-LCoS devices in a wide variety of working conditions ${ }^{[19]}$. This predictive capability was at first demonstrated in polarimetric applications addressing uniform screens onto the PA-LCoS microdisplay ${ }^{[18]-[20]}$. Recently ${ }^{[21]}$ we also demonstrated that performance of non-uniform phase-only elements can also be predicted with our methodology. It comprises both a time-dependent model for the retardance fluctuations and a reverse-engineering approach to calibrate the value of the parameters in the model. The calibration is performed by applying uniform gray level screens onto the

Optics and Photonics for Information Processing IX, edited by Abdul A. S. Awwal, Khan M. Iftekharuddin,

Mohammad A. Matin, Mireya García Vázquez, Andrés Márquez, Proc. of SPIE Vol. 9598, 95980C

(C) 2015 SPIE · CCC code: $0277-786 X / 15 / \$ 18 \cdot$ doi: $10.1117 / 12.2188039$

Proc. of SPIE Vol. 9598 95980C-1 
PA-LCoS device. It has been very important to verify that these calibrated values can also be employed to predict the performance when non-uniform screens are addressed. In particular, we considered ${ }^{[21]}$ blazed gratings with a $2 \pi$ radians phase depth, with different spatial periodicities and with a different number of quantization levels. Specifically we applied one pixel per level, so that an N-levels blazed grating has an N-pixels period. Both the static performance and the dynamic variation of the diffraction efficiencies were analyzed.

In the paper we take advantage of the demonstrated predictive capability of our approach to analyse further the effect of flicker in the performance of blazed gratings in a wide set of conditions. In particular in this work we are interested in the analysis of the diffraction efficiencies, both the static and the dynamic performance, for the zero and first order as a function of the magnitude of the phase flicker to find the range of applicability of PA-LCoS devices in applications.

\section{PA-LCOS: MODEL AND CALIBRATION}

Let us first introduce the basics of our theoretical approach, which is composed of both a model for the time evolution of the retardance fluctuations in PA-LC devices, and reverse-engineering calibration procedure to extract the value of the parameters in the model. The phase flicker exhibited by LCoS devices within the frame period has been found to be properly approximated by a triangular time-dependent profili ${ }^{[13][18]}$. If we consider the averaged value for a measurement integration time equal or larger than the addressing frame period what we get is that the state of polarization (SOP) for the light beam reflected by the LCoS may not be fully polarized. This leads us to use the Mueller-Stokes formalism, which enables to deal both with polarized and unpolarized light ${ }^{[22]}$, to describe the action of PA-LCoS displays on the light beam. Applying this formalism and taking into account the triangular time-dependent profile for the linear retardance, we obtained the following matrix for a linear variable retarder with flicker,

$$
\left\langle M_{R}(\bar{\Gamma}, a)\right\rangle=\left(\begin{array}{cccc}
1 & 0 & 0 & 0 \\
0 & 1 & 0 & 0 \\
0 & 0 & (\sin a / a) \cos \bar{\Gamma} & (\sin a / a) \sin \bar{\Gamma} \\
0 & 0 & -(\sin a / a) \sin \bar{\Gamma} & (\sin a / a) \cos \bar{\Gamma}
\end{array}\right)
$$

, where $\bar{\Gamma}$ is the average retardance within the frame period and $a$ its fluctuation amplitude, defined as half the maximum-to-minimum value for the fluctuation. To calibrate the values for these two parameters in the model we measured the averaged Stokes vector parameters for an input light beam linearly polarized light at $+45^{\circ}$ with respect to the $\mathrm{X}$ axis, i.e. $(\mathrm{S} 0=1, \mathrm{~S} 1=0, \mathrm{~S} 2=1, \mathrm{~S} 3=0)$. The analytical expressions for the reflected averaged Stokes vector and for the degree of polarization (DoP) are very simple in this case,

$$
\begin{gathered}
\left\langle S_{\text {out }}\right\rangle=\left(\begin{array}{c}
1 \\
0 \\
-(\sin a / a) \cos \bar{\Gamma} \\
(\sin a / a) \sin \bar{\Gamma}
\end{array}\right) \\
D o P=(\sin a / a)
\end{gathered}
$$

Eventually, the calibration can be easily accomplished using Eq. (3) to obtain the fluctuation amplitude $a$, and the ratio between the 3rd and 4th Stokes vector components, i.e. $-\left\langle S_{3}\right\rangle /\left\langle S_{2}\right\rangle=\operatorname{tg}(\bar{\Gamma})$, to obtain $\bar{\Gamma}^{[18]}$.

The PA-LC device that we consider is a commercial PA-LCoS display, model PLUTO distributed by the company HOLOEYE. It is filled with a nematic liquid crystal, with 1920x1080 pixels and 0.7" diagonal, $8 \mu \mathrm{m}$ pixel pitch, $83 \%$ fill factor, and digitally addressed. By means of a RS-232 interface and its correspondingly provided software, we can configure the modulator for different applications and wavelengths. Besides, different pulse width modulation (PWM) addressing schemes (digital addressing sequences) can be generated by the driver electronics ${ }^{[23][24]}$. In this work we consider the electrical sequence labeled as "18-6 633 2pi linear". The actual number of quantization values available with this configuration file is 256 . 
The working geometry considered in this paper corresponds to quasi-perpendicular incidence ${ }^{[19]}$ (actual angle of incidence is $3^{\circ}$ ). We note that the director axis (extraordinary axis) in nematic based LCoS generally corresponds to the slow axis. In the present $\mathrm{LCOS}$ the director axis is along the horizontal. In Fig. 1 we show the calibrated values for the average retardance and its flicker amplitude versus the gray level addressed (voltage). We observe that the retardance range is about $360^{\circ}$ with a very good linearity. The fluctuation amplitude reaches $50^{\circ}$ with its maximum value achieved about gray level 200. We note that for $\mathrm{DoP}>1$, non-physical values, we consider that fluctuation amplitude is $0^{\circ}$, as can be seen for low gray level values ${ }^{[18]}$. The standard deviation (or root mean square) calculated from the values of the fluctuation amplitude across the whole gray level range is equal to $37^{\circ}$. We will call this magnitude the flicker standard deviation and it will be used extensively as a figure of merit to compare the severity of the phase flicker when simulating the various blazed gratings.

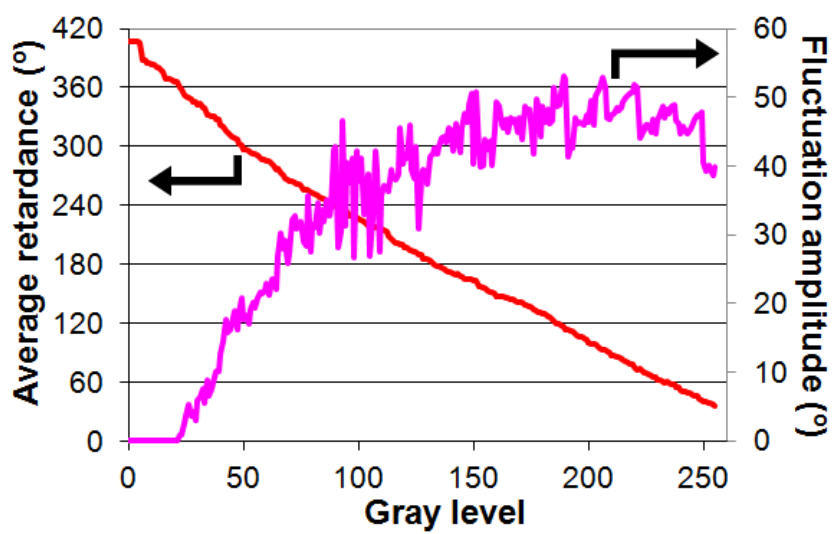

Fig. 1. Calculated values for average retardance and fluctuation amplitude corresponding to sequence " $18-6633$ 2pi linear" for $\lambda=633 \mathrm{~nm}$, and for quasi-perpendicular incidence at $3^{\circ}$.

\section{BLAZED GRATINGS: EXPERIMENT}

In the experiment with the blazed gratings we follow the same procedure as in the previous paper ${ }^{[21]}$, where we considered a series of blazed gratings with different number of quantization levels and with one pixel per level. Due to the pixelated structure of LCoS devices this translates into different spatial periodicities in the experiment. The gratings are considered to have a $360^{\circ}$ phase depth ${ }^{[25]}$, so that maximum diffraction efficiency in the first order can be reached, which can be analytically calculated with the following expression ${ }^{[4]}$,

$$
\eta_{1}=(\sin (\pi / q) /(\pi / q))^{2}
$$

, where $q$ is the number of quantization levels. For example $\eta_{1}=0.987$ for $q=16$ levels, i.e. basically performs as a continuous profile. On the lower diffraction efficiency limit, $\eta_{1}=0.405$ for $q=2$ levels, which actually corresponds to a binary phase grating with a $180^{\circ}$ phase depth. We also note that for a $360^{\circ}$ phase depth blazed grating the zero order diffraction efficiency is $\eta_{0}=0$. As an additional remark, in the simulations in the present work, as in ${ }^{[21]}$, even though the calibration curve for the retardance in Fig. 1 shows a good linearity, we apply the appropriate linearization look-up table, so that the residual non-linearity is compensated via software.

For the experiments in the lab, a light beam from a He-Ne laser at $633 \mathrm{~nm}$ is expanded using a spatial filter and then is collimated with a lens. The angle of incidence onto the $\operatorname{LCoS}$ is $3^{\circ}$, which corresponds to the calibration conditions for the results in Fig. 1. The collimated beam of light passes through a polarizer whose transmission axis is oriented so that the incident SOP corresponds to linearly polarized light along the lab-horizontal, i.e. parallel to the director axis of the liquid crystal in the LCoS: this is the phase-only configuration. We have checked that there is no need for an analyzer at the output since the reflected SOP is fully linearly polarized along the lab-horizontal. A lens focuses the reflected beam to its focal plane where a detector connected to a radiometer measures the diffracted intensity. 
We note that due to the pixelated structure of the device multiple replicas of the diffraction orders of the blazed grating are generated, each replica centered at the position of one of the diffraction orders produced by the periodic pixelated structure. This is a well known consequence of the sampling theory ${ }^{[26]}$. We restrict our attention to the zero order replica, i.e. the one centered at the position of the zero order produced by the periodic pixelated structure. In principle the relative energy deflected to each of the replicas does not depend on the image being addressed but only on geometrical characteristics of the display, basically on the fill factor. However, during the realization of the experiment in the lab we observed a very clear change of brightness between different replicas when a uniform screen was sent with respect to sending the blazed gratings. As we explained in $^{[21]}$, probably the different exchange of energy between replicas when changing the image being addressed onto the LCoS is due to the pixel crosstalk caused by the fringing fields, i.e. the gradual voltage changes across the border of neighboring pixels, and by elastic forces in the LC material preventing abrupt spatial variations in the phase modulation ${ }^{[27][28]}$. Both effects cause the realized phase modulation of a pixel to depend also on the voltage applied over adjacent pixels. This cross talk phenomenon increases as pixels get smaller and as voltage changes between neighbouring pixels are larger. We applied ${ }^{[21]}$ a renormalization factor to compensate for this effect in the measurements since we were specifically interested in the analysis of phase flicker effects.

In Fig. 2 we show for the sequence "18-6 633 2pi linear" the results for the measurements of the instantaneous first order diffraction efficiencies for 6 blazed gratings. Next to each curve we show the quantization number of levels, $q$, in each case. All the gratings are measured and optimized for the wavelength $633 \mathrm{~nm}$. The instantaneous values have been obtained by connecting a radiometer model $1830-\mathrm{C}$ from Newport, which has an analog bandwidth greater than $30 \mathrm{kHz}$, to a digital oscilloscope model TDS-1012b from Tektronix, with a $100 \mathrm{MHz}$ analog bandwidth. First thing we note is that the diffracted intensity fluctuates, with a period of $8.33 \mathrm{~ms}$, i.e. frequency $120 \mathrm{~Hz}$. In these results the amplitude of fluctuations becomes smaller as the number of quantization levels increases. Additionally, the average diffraction also increases with $q$ which is the theoretically expected result.

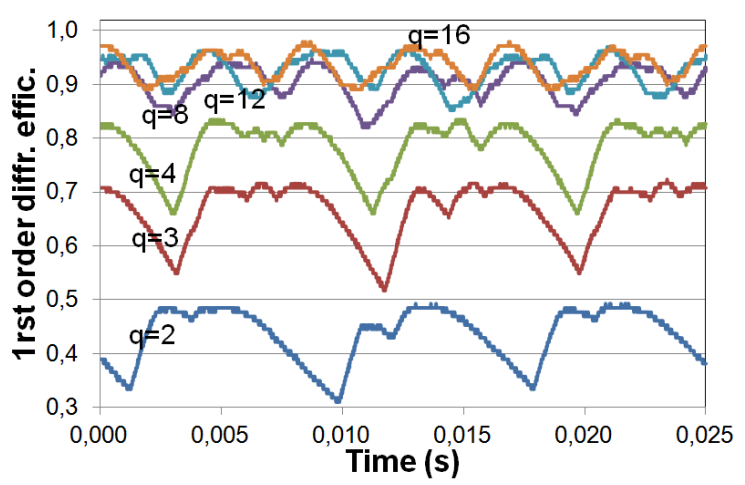

Fig. 2. Instantaneous values for the first order diffracted intensity for blazed gratings with different quantization levels $(q$ shown on the various curves), corresponding to sequence " $18-66332$ pi linear" for $\lambda=633 \mathrm{~nm}$, and for quasi-perpendicular incidence at $3^{\circ}$.

\section{SIMULATION OF FLICKER EFFECTS IN PHASE MULTILEVEL ELEMENTS}

The procedure that we follow for the simulation is the one described in a previous paper ${ }^{[21]}$. First we calculate the gray level $g$ to be applied to produce the desired phase value $\varphi$ at each step in the multilevel blazed grating. To this goal we consider the calibrated values $\bar{\Gamma}(g)$ given in Fig. 1, and for each desired $\varphi$ we look for the closest available average retardance value $\bar{\Gamma}$ : closest in terms of the euclidean distance in the complex plane between the two values ${ }^{[25]}$. Second, the frame period $\mathrm{T}$ is divided in time intervals small enough so that the fluctuating phase profile of the blazed grating is accurately sampled. At each time sample, the staircase phase profile is partly distorted from the ideal quantized linear ramp since each step, associated with a specific gray level $g$, is fluctuating with a different amplitude $a(g)$, given in Fig. 1. The various phase steps in the staircase profile fluctuate in a correlated form with each other, in accordance with the periodic behavior exhibited by the instantaneous values in Fig. 2. As a starting value, at time $\mathrm{t}=0$ we have chosen for each phase step $\varphi$ the phase value $\bar{\Gamma}(g)-a(g)$ which then increases linearly with time till $\bar{\Gamma}(g)+a(g)$. Another 
starting set of values may be chosen, resulting in different instantaneous profiles, but since we are interested in averaged effects this has no relevance on the results. As a third and final part of the simulation, at each time sample the Fourier transform of the blazed grating profile is calculated: this provides the instantaneous diffraction efficiency. From these instantaneous values we calculate the averaged one and the amplitude and standard deviation for its fluctuation.

In the previous paper ${ }^{[21]}$ the numerically calculated values were compared with experimental measurements showing a very good agreement. This validation of the theoretical approach enables us in the present work to explore further, through simulation, the effects of flicker on the performance of multilevel phase elements, and specifically on blazed gratings. In this Section we will present numerical results dealing both with the average diffraction efficiency, i.e. static analysis, and with its associated fluctuation, i.e. dynamic analysis. We concentrate on learning what are the tendencies exhibited both in the static and dynamic analysis as a function of the degree of the fluctuations in the retardance. We run the simulations for three different numbers of quantization levels $q$ in the blazed gratings, equal to 2, 8 and 16 levels. They span from the lowest number, 2, to 16 levels, which is close enough to a continuous profile in terms of diffraction efficiency. To simulate different magnitudes of fluctuation in the retardance we consider the flicker versus gray level profile in Fig. 1, and this flicker profile is multiplied for a varying scale factor ranging from 0 to 2.2, i.e. the flicker amplitude range covers from the non-fluctuations case to the case where the amplitude of the fluctuations is slightly larger than $100^{\circ}$. In terms of the flicker standard deviation (std. dev.) this is equivalent to a range from $0^{\circ}$ to about $80^{\circ}$. At a flicker std. dev. of $37^{\circ}$ the flicker profile corresponds to the one in Fig. 1, i.e. the scale factor applied is 1.

First, we will show the results obtained with the static analysis, where the magnitude of interest is the average diffraction efficiency. In Fig. 3(a) and (b) we show the diffraction efficiency for the zero and for the first order respectively as a function of the standard deviation of the flicker retardance, parameter described in the previous paragraph. On the plots we have labeled the number of quantization levels $(2,8$ and 16) for the three gratings considered. In Fig. 3(a) we observe that the energy diffracted into the zero order increases with the increase in the retardance flicker. Numerical fitting shows that this increase follows a parabolic behavior. An important result is that the zero order magnitude diminishes dramatically as the number of quantization levels increase. This is interesting since in many applications in digital holography or in diffractive optics, and probably in other areas as well, we need the zero order to remain as low as possible since it overlaps with the desired signal, as it may happen for example when displaying diffractive lenses or kinoforms ${ }^{[4]}$. In Fig. 3(b) the diffraction efficiency of the first order decreases with the increase in the retardance flicker and we have verified that it follows a non-linear parabolic behavior. This means that for low flicker values the deviation from the ideal case with no fluctuations is still very small. We note how close to one is the diffraction efficiency for the cases with 8 and 16 quantization levels.
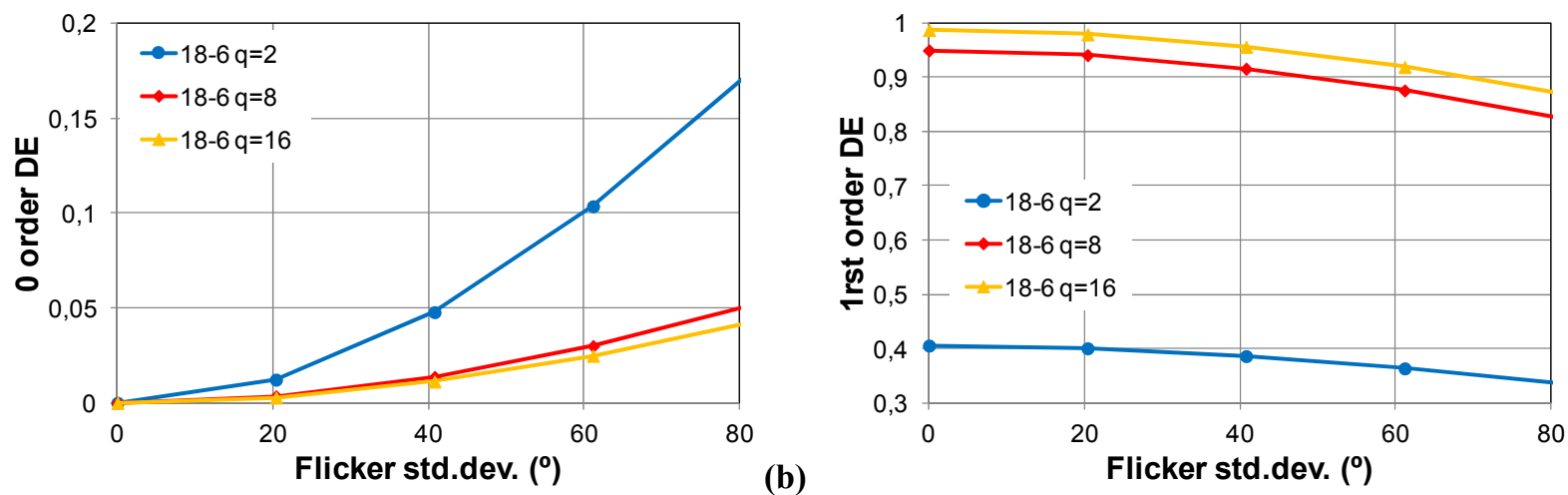

(a)

(b)

Flicker std.dev. $\left({ }^{\circ}\right)$

Fig. 3. Realistic simulations for the diffraction efficiency. (a) Zero order; (b) First order. Sequence "18-6 633 2pi linear" for

$\lambda=633 \mathrm{~nm}$, and for quasi-perpendicular incidence at $3^{\circ}$.

In Fig. 4 we represent for the first diffraction order the deviation between the ideal diffraction efficiency (i.e. non-flicker case) and the realistic (taking into account the flicker), normalized by the ideal diffraction efficiency. We see that the normalized difference increases with the flicker increase. However the diminution in diffraction efficiency is still very low, about $5 \%$, at a flicker std. dev. of $40^{\circ}$, for the binary grating. And it is even smaller as the number of quantization levels increase. This helps us to realize to which point the flicker is actually not a limitation in many applications especially if the number of quantization levels is large enough. 


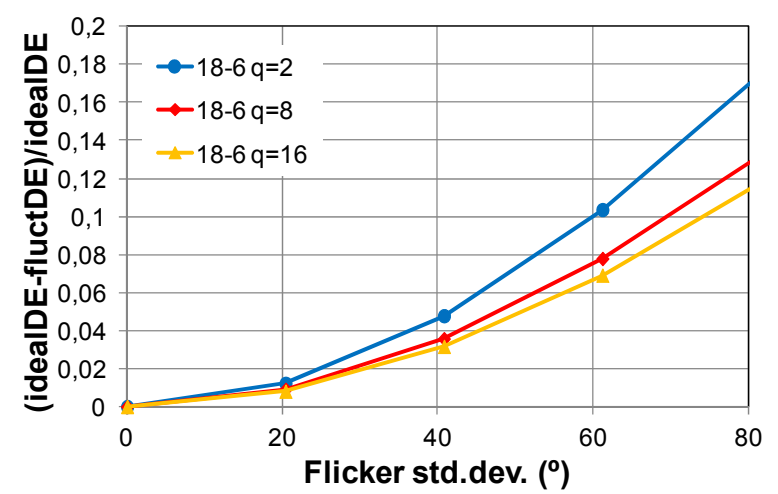

Fig. 4. For the first order diffraction efficiency, deviation between the ideal (i.e. non-flicker) and the realistic numerical simulation (i.e. including the calibrated fluctuations), normalized by the ideal diffraction efficiency. Sequence "18-6 633 2pi linear" for $\lambda=633 \mathrm{~nm}$, and for quasi-perpendicular incidence at $3^{\circ}$.

Next we show the results dealing with the dynamic analysis, where the magnitude of interest is the standard deviation value of the fluctuations for the zero and for the first diffraction orders. This is what we show respectively in Fig. 5(a) and (b) as a function of the standard deviation of the flicker retardance. We see in Fig. 5(a) that fluctuations for the zero order are larger for lower number of quantization levels. The increase in the retardance flicker increases parabolically the fluctuations in the zero order. In Fig. 5(b), for the first diffraction order, we see that the level of fluctuations is larger for the gratings with 8 and 16 quantization levels. This does not agree with the result in Fig. 2, where the fluctuations were actually larger for the binary grating, i.e. $q=2$. We do not have a clear explanation for this difference between experiment and simulation. It could be that the experimental measurements in Fig. 2, for the binary grating, were partly affected by the aliasing from the diffracted order in the first replica produced by the pixilation of the LCoS display. This aliasing cannot be avoided in the experiment.
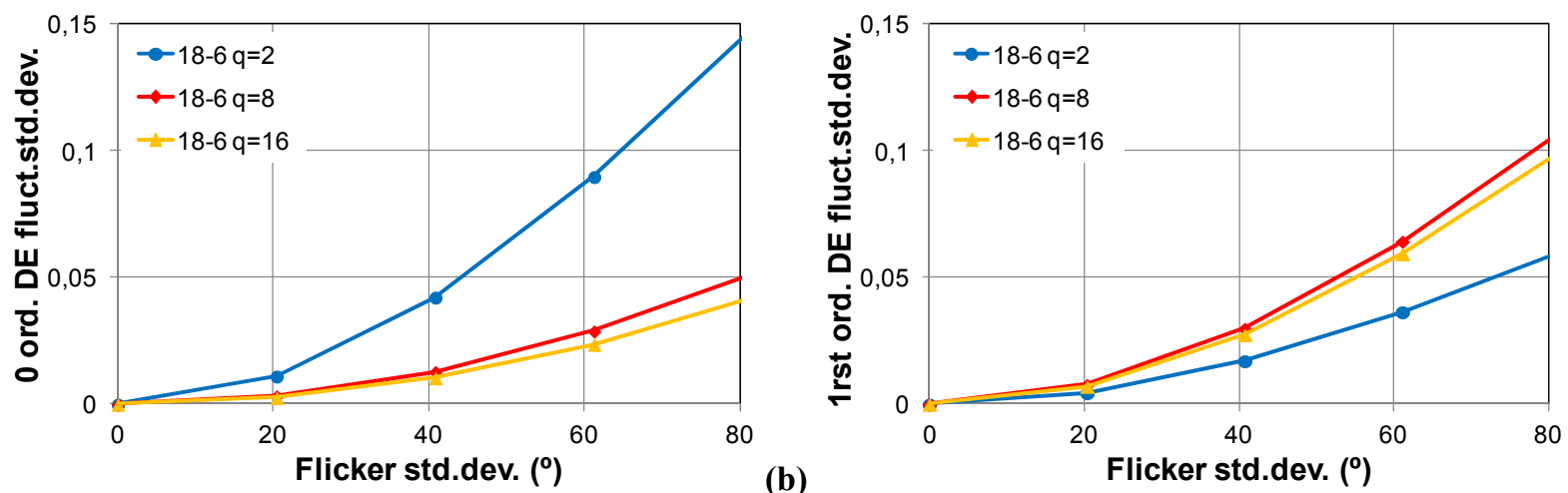

Fig. 5. Standard deviation of the time fluctuation for the diffraction efficiency. (a) Zero order; (b) First order. Sequence "1866332 pi linear" for $\lambda=633 \mathrm{~nm}$, and for quasi-perpendicular incidence at $3^{\circ}$.

In Fig. 6 we show the standard deviation of the fluctuations for the first order normalized by its average diffraction efficiency. The larger relative fluctuations correspond to the binary grating, and they decrease with the increase in the number of quantization levels. Values are smaller than a 5\% within the range of retardance flicker standard deviation smaller than $40^{\circ}$. 


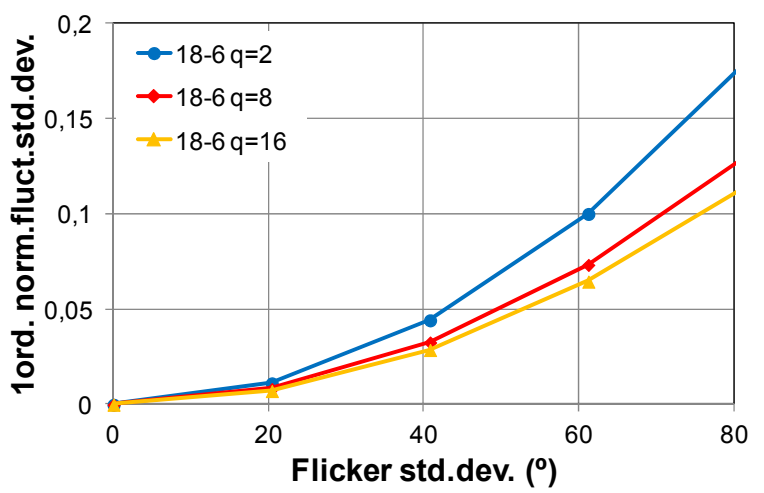

Fig. 6. For the first diffraction order, standard deviation of the time fluctuation normalized by its average value. Sequence "18-6 6332 pi linear" for $\lambda=633 \mathrm{~nm}$, and for quasi-perpendicular incidence at $3^{\circ}$.

\section{CONCLUSIONS}

In a previous paper ${ }^{[21]}$ we validated a theoretical approach, based on a time averaged Stokes polarimetric calibration procedure, to predict the performance of multilevel phase elements. This validation of the theoretical approach has enabled us in the present work to explore further, through simulation, the effects of flicker on the performance of multilevel phase elements, and specifically on blazed gratings. We have presented numerical results dealing both with the average diffraction efficiency, i.e. static analysis, and with its associated fluctuation, i.e. dynamic analysis. We concentrate on learning what are the tendencies exhibited both in the static and dynamic analysis as a function of the degree of the fluctuations in the retardance. We run the simulations for three different numbers of quantization levels $q$ in the blazed gratings, equal to 2, 8 and 16 levels. As a general result we obtained that the various magnitudes, diffraction efficiencies and standard deviation of the fluctuation, both absolute and normalized, show a parabolic variation with the retardance flicker. For values of the standard deviation of the retardance flicker smaller than $40^{\circ}$, the values in most of the figures of merit are within a 5\% with respect to the case with no flicker, and if we consider a retardance flicker smaller than $20^{\circ}$, then the values of the figures of merit are within a $2^{\circ}$. These are realizable retardardance flicker values as shown in paper ${ }^{[21]}$. Thus, degradation due to flicker can be very low and within the tolerance range for many applications. Further, an important result obtained is that performance gets better with the increase in the number of quantization levels in the element. This may produce phase elements basically unaffected by the retardance flicker.

\section{ACKNOWLEDGEMENTS}

Work supported by the Ministerio de Trabajo y Competitividad of Spain under projects FIS2011-29803-C02-01 and FIS2011-29803-C02-02 and by the Generalitat Valenciana of Spain (projects PROMETEOII/2015/015 and ISIC/2012/013).

\section{REFERENCES}

[1] N. Collings, T. Davey, J. Christmas, D. Chu, and B. Crossland, "The Applications and Technology of PhaseOnly Liquid Crystal on Silicon Devices," J. Display Technol. 7, 112-119 (2011).

[2] G. Lazarev, A. Hermerschmidt, S. Krüger, and S. Osten, "LCOS Spatial Light Modulators: Trends and Applications," in [Optical Imaging and Metrology: Advanced Technologies], W. Osten and N. Reingand, eds., (John Wiley \& Sons, 2012).

[3] Z. Zhang, Z. You, and D. Chu, "Fundamentals of phase-only liquid crystal on silicon (LCOS) devices," Light Sci. Appl. 3, 1-10 (2014).

[4] J. Turunen and F. Wyrowski, eds., [Diffractive Optics for Industrial and Commercial Applications], Akademie Verlag (1997).

[5] H. J. Coufal, D. Psaltis, and B. T. Sincerbox, eds., [Holographic Data Storage], Springer-Verlag (2000). 
[6] K. Curtis, L. Dhar, A. Hill, W. Wilson, and M. Ayres, eds., [Holographic Data Storage: From Theory to Practical Systems], John Wiley \& Sons, Ltd, (2010).

[7] W. Osten, C. Kohler, and J. Liesener, "Evaluation and application of spatial light modulators for optical metrology," Opt. Pura Apl. 38, 71-81 (2005).

[8] M. A. F. Roelens, S. Frisken, J. A. Bolger, D. Abakoumov, G. Baxter, S. Poole, and B. J. Eggleton, "Dispersion trimming in a reconfigurable wavelength selective switch," J. Lightw. Technol. 26, 73-78 (2008).

[9] M. Salsi, C. Koebele, D. Sperti, P. Tran, H. Mardoyan, P. Brindel, S. Bigo, A. Boutin, F. Verluise, P. Sillard, M. Bigot-Astruc, L. Provost, and G. Charlet, "Mode-Division Multiplexing of $2100 \mathrm{~Gb} / \mathrm{s}$ Channels Using an LCOS-Based Spatial Modulator," J. Lightwave Technol. 30, 618-623 (2012).

[10] A. Dudley, G. Milione, R. R. Alfano, and A. Forbes, "All-digital wavefront sensing for structured light beams," Opt. Express 22, 14032-14040 (2014).

[11] A. Farré, M. Shayegan, C. López-Quesada, G. A. Blab, M. Montes-Usategui, N. R. Forde, E. Martín-Badosa, "Positional stability of holographic optical traps," Opt. Express 19, 21370-21384 (2011).

[12] M. A. Solís-Prosser, A. Arias, J. J. M. Varga, L. Rebón, S. Ledesma, C. Iemmi, and L. Neves, "Preparing arbitrary pure states of spatial qudits with a single phase-only spatial light modulator," Opt. Lett. 38, 4762-4765 (2013).

[13] A. Lizana, I. Moreno, A. Márquez, C. Iemmi, E. Fernández, J. Campos, and M. J. Yzuel, "Time fluctuations of the phase modulation in a liquid crystal on silicon display: characterization and effects in diffractive optics," Opt. Express 16, 16711-16722 (2008).

[14] A. Lizana, I. Moreno, A. Márquez, E. Also, C. Iemmi, J. Campos, and M.J.Yzuel, "Influence of the temporal fluctuations phenomena on the ECB LCoS performance," Proc. SPIE 7442, 74420G-1 (2009).

[15] J. García-Márquez, V. López, A. González-Vega, and E. Noé, "Flicker minimization in an LCoS spatial light Modulator," Opt. Express 20, 8431-8441 (2012).

[16]F. J. Martínez, A. Márquez, S. Gallego, J. Francés, and I. Pascual, "Extended linear polarimeter to measure retardance and flicker: application to LCoS devices in two working geometries," Opt. Eng. 53, 014105 (2014).

[17]C. Ramirez, B. Karakus, A. Lizana, and J. Campos, "Polarimetric method for liquid crystal displays characterization in presence of phase fluctuations," Opt. Express 21, 3182-3192 (2013).

[18]F.J. Martínez, A. Márquez, S. Gallego, J. Francés, I. Pascual, and A. Beléndez, "Retardance and flicker modeling and characterization of electro-optic linear retarders by averaged Stokes polarimetry," Opt. Lett. 39, 1011-1014 (2014).

[19]F.J. Martínez, A. Márquez, S. Gallego, M. Ortuño, J. Francés, A. Beléndez, and I. Pascual, "Averaged Stokes polarimetry applied to evaluate retardance and flicker in PA-LCoS devices," Opt. Express 22, 15064-15074 (2014).

[20] A. Márquez, F. J. Martínez, S. Gallego, M. Ortuño, J. Francés, A. Beléndez, and I. Pascual, “Averaged Stokes polarimetry applied to characterize parallel-aligned liquid crystal on silicon displays," Proc. SPIE 9216, 9216H (2014).

[21]F.J. Martínez, A. Márquez, S. Gallego, M. Ortuño, J. Francés, I. Pascual, and A. Beléndez, "Predictive capability of average Stokes polarimetry for simulation of phase multilevel elements onto LCoS devices," Appl. Opt. 54, 1379-1386 (2015).

[22] G. Goldstein, [Polarized Light], Marcel Dekker (2003).

[23] A. Hermerschmidt, S. Osten, S. Krüger, and Thomas Blümel, "Wave front generation using a phase-only modulating liquid-crystal based micro-display with HDTV resolution," Proc. SPIE 6584, 65840E (2007).

[24]F. J. Martínez, A. Márquez, S. Gallego, M. Ortuño, J. Francés, A. Beléndez, and I. Pascual, "Electrical dependencies of optical modulation capabilities in digitally addressed parallel aligned LCoS devices," Opt. Eng. 53, 067104 (2014).

[25] A. Lizana, A. Márquez, L. Lobato, Y. Rodange, I. Moreno, C. Iemmi, and J. Campos, "The minimum Euclidean distance principle applied to improve the modulation diffraction efficiency in digitally controlled spatial light modulators," Opt. Express 18, 10581-10593 (2010).

[26] J. W. Goodmann, [Introduction to Fourier-Optics, 3rd Edition], Roberts \& Company (2005).

[27] B. Apter, U. Efron, and E. Bahat-Treidel, "On the fringing-field effect in liquid-crystal beam-steering devices," Appl. Opt. 43, 11-19 (2004).

[28]C. Lingel, T. Haist, and W. Osten, "Optimizing the diffraction efficiency of SLM-based holography with respect to the fringing field effect," Appl. Opt. 52, 6877-6883 (2013). 